Author affiliations appear at the end of this article.

Published at ascopubs.org/journal/jco on October 31, 2016

Support information appears at the end of this article.

Clinical trial information: NCT00949455.

Corresponding author: Tom Powles, MRCP, MD, Experimental Cancer Medicine Centre, Barts Cancer Institute, Barts Health NHS Trust and the Royal Free NHS Trust, Queen Mary University of London, London EC1A 7BE, United Kingdom; e-mail: thomas.powles@ bartshealth.nhs.uk

(C) 2016 by American Society of Clinical Oncology

0732-183X/17/3501w-48w/\$20.00

\title{
Phase III, Double-Blind, Randomized Trial That Compared Maintenance Lapatinib Versus Placebo After First-Line Chemotherapy in Patients With Human Epidermal Growth Factor Receptor 1/2-Positive Metastatic Bladder Cancer
}

Thomas Powles, Robert A. Huddart, Tony Elliott, Shah-Jalal Sarker, Charlotte Ackerman, Robert Jones, Syed Hussain, Simon Crabb, Satinder Jagdev, John Chester, Serena Hilman, Mark Beresford, Graham Macdonald, Sundar Santhanam, John A. Frew, Andrew Stockdale, Simon Hughes, Daniel Berney, and Simon Chowdhury

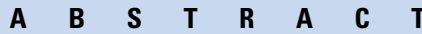

\section{Purpose}

To establish whether maintenance lapatinib after first-line chemotherapy is beneficial in human epidermal growth factor receptor (HER) 1/HER2-positive metastatic urothelial bladder cancer (UBC).

\section{Methods}

Patients with metastatic UBC were screened centrally for HER1/HER2 overexpression. Patients who screened positive for HER1/2 and who did not have progressive disease during chemotherapy (four to eight cycles) were randomly assigned one to one to lapatinib or placebo after completion of first-line/initial chemotherapy for metastatic disease. The primary end point was progression-free survival (PFS).

\section{Results}

Between 2007 and 2013, 446 patients with UBC were screened, and 232 with HER1- or HER2positive disease were randomly assigned. The median PFS for lapatinib and placebo was 4.5 (95\% $\mathrm{Cl}, 2.8$ to 5.4 ) and $5.1(95 \% \mathrm{Cl}, 3.0$ to 5.8) months, respectively (hazard ratio, $1.07 ; 95 \% \mathrm{Cl}, 0.81$ to $1.43 ; P=.63)$. The overall survival for lapatinib and placebo was $12.6(95 \% \mathrm{Cl}, 9.0$ to 16.2$)$ and 12.0 $(95 \% \mathrm{Cl}, 10.5$ to 14.9$)$ months, respectively (hazard ratio, $0.96 ; 95 \% \mathrm{Cl}, 0.70$ to $1.31 ; P=.80$ ). Discontinuation due to adverse events were similar in both arms (6\% lapatinib and $5 \%$ placebo). The rate of grade 3 to 4 adverse events for lapatinib and placebo was $8.6 \%$ versus $8.1 \%(P=.82)$. Preplanned subset analysis of patients strongly positive for HER1/HER2 (3+ on immunohistochemistry; $n=111)$, patients positive for only HER1 $(n=102)$, and patients positive for only HER2 $(n=42)$ showed no significant benefit with lapatinib in terms of PFS and overall survival $(P>.05$ for each).

\section{Conclusion}

This trial did not find significant improvements in outcome by the addition of maintenance lapatinib to standard of care.

\section{J Clin Oncol 35:48-55. (C) 2016 by American Society of Clinical Oncology}

\section{INTRODUCTION}

The overall survival (OS) of patients with metastatic urothelial bladder cancer (UBC), also known as transitional cell cancer (TCC), is short. Treatment of metastatic disease focuses on platinumbased combination chemotherapy in the first-line setting. ${ }^{1,2}$ After chemotherapy is complete, patients undergo a period of observation. The majority of these patients experience a relapse and die as a result of the disease. Further, second-line chemotherapy at this point remains controversial, with no clear survival advantage. ${ }^{3}$

To date, the Food and Drug Administration has not approved targeted treatments for metastatic UBC despite a number of molecular targets, such as the human epidermal growth factor receptor (HER) family and vascular endothelial growth factor, that appear attractive preclinically. ${ }^{4-6}$ Clinical studies that tested these agents in unselected patients failed to reproduce this in vivo activity. ${ }^{7-9}$ Three possible reasons account for these results. First, the combination of chemotherapy and targeted 
therapy in the UBC population, which has multiple comorbidities, is difficult. ${ }^{7,8,10,11}$ Second, none of the randomized phase III trials to date have selected patients based on expression of molecular targets. Finally, UBC has a high frequency of mutations, therefore the targeting of only one protein may be inadequate to achieve clinical benefit. $^{12}$

To address these issues, the UK Bladder Cancer Clinical Studies Group embarked on a phase III randomized trial to test single lapatinib (an HER1 and HER2 tyrosine kinase inhibitor) against placebo in HER1- or HER2-positive advanced/metastatic UBC. The drug was tested in the period after completion of first-line chemotherapy for metastatic disease with the primary aim of delaying progression-free survival (PFS). The goal was to maintain the response to chemotherapy; hence, the term maintenance therapy. Placebo was used as the control to allow for double blinding.

Lapatinib was chosen as the study drug because it targets HER1 and HER2, both of which have been implicated in bladder cancer progression. ${ }^{5,6,13,14}$ Preclinical and phase II data support its use in selected patients with HER1- or HER2positive cancer (on immunohistochemistry [IHC]). ${ }^{15}$ In addition, as a single agent, lapatinib appears to be well tolerated, which is important in this population where comorbidities are common.

\section{METHODS}

\section{Screening Phase}

Eligible patients were those with a component of histologically confirmed advanced/metastatic TCC of the urothelial tract. Details of the percentage of TCC histology were not collected. Archived paraffinembedded tissue was used. There was no limit on the age of the sample. Sites sent the most recent sample for testing when multiple samples were available from the same patient. Screening occurred during or after the completion of first-line chemotherapy for metastatic disease. Pathology samples were centrally tested for HER1 and HER2. Patients with positive results were eligible to participate in the trial. Baseline characteristics, treatment, and outcome data were collected for the entire screened population to assess prognostic factors. All patients gave informed consent for this trial, which has appropriate ethical approval.

\section{HER1 and HER2 Testing}

Overexpression of HER1 and HER2 was performed by using IHC and fluorescent in situ hybridization (FISH). IHC was performed by using the avidin-biotinylated peroxidase complex staining method standardized for both antibodies. ${ }^{16}$ The primary antibody was incubated for $1 \mathrm{~h}$ per the optimized method for each antibody (Novocastra antibodies HER1 [NCL-EGFR] 1:20 and HER2 [NCL-CBE-356] 1:80; Leica Biosystems Newcastle Ltd, Newcastle Upon Tyne, United Kingdom). IHC scoring was performed independently and blinded by a single pathologist to allow for rapid turnaround of samples. Independent double biomarker assessment would have been preferable, but it was not logistically possible in this study. Expression was scored by staining intensity (0, negative; $1+$ weakly positive; $2+$, moderately positive; $3+$, strongly positive). ${ }^{17}$ Only patients with $2+$ or $3+$ on IHC for HER1 and/or HER2 were considered to be have a positive finding and were eligible for the study. ${ }^{18}$ FISH was performed in patients with equivocal positivity $(1+$ on IHC with both antibodies), and all had negative results. This method has been used previously to test HER1 and HER2 status in lapatinib trials and was deemed the most appropriate at the time of trial inception. ${ }^{15,16}$

\section{Key Eligibility Criteria}

Patients were required to have completed four to eight cycles of chemotherapy for advanced metastatic UBC. Random assignment needed to occur between 4 and 10 weeks after the completion of chemotherapy. Any recognized chemotherapy regimen for metastatic UBC was permitted. Prior adjuvant or neoadjuvant chemotherapy was not considered first-line chemotherapy. Patients with radiologic progression of disease during chemotherapy were excluded. Adequate renal, hematologic, and liver function were required. Patients with a left ventricular ejection fraction (LVEF) below the normal range were excluded. Patients were required to be at least 18 years of age and to have resolution of chemotherapy-related toxicity before random assignment.

\section{Evaluation on Study}

Before random assignment, patient history, examination, trial-related blood tests, and cross-sectional imaging occurred. Adverse events (AEs) were graded according to the Common Toxicity Criteria for Adverse Events (version 3). Disease status and LVEF were assessed every 12 weeks. Response and progression were assessed by Response Evaluation Criteria in Solid Tumors (RECIST; version 1.1). No central review occurred. Patients were discontinued from the study at progression, withdrawal of consent, unacceptable toxicity, or death.

\section{Treatment Plan}

Patients were randomly assigned in a double-blind manner to lapatinib or placebo (1:1). Stratification occurred by prior response to first-line chemotherapy (stable disease versus partial response/complete response) and Eastern Cooperative Oncology Group performance status. Lapatinib was administered continuously at $1,500 \mathrm{mg}$ once daily (six 250-mg tablets). In the placebo group six visually identical tablets were administered instead. Dose reductions to five (lapatinib $=1,250 \mathrm{mg}$ ) or four (lapatinib $=1,000 \mathrm{mg}$ ) tablets could occur based on AEs outlined in the protocol (Data Supplement).

\section{End Points and Statistical Considerations}

The primary end point was PFS from the time of random assignment to progression or death. All randomly assigned patients were included in the analysis (Fig 1). This design was intention to treat. Screening for HER1 and HER2 status occurred before study entry (screening population). Secondary end points were OS; response rates; AEs; and outcome of subsets, which depended on biomarker status (HER1 or HER2 positive alone or HER1 and HER2 3+ IHC).

The phase III study required approximately 221 patients for 196 events on the basis of a single-sided design with alpha $=.025$ to detect a $60 \%$ longer median PFS (hazard ratio [HR], 0.62) in the treatment group with $90 \%$ power. The duration of this PFS was assumed to be 6 months, although there was a lack of previous data to guide this estimation.

The trial followed a phase III continuous design with interim analyses. An independent data monitoring committee assessed efficacy and toxicity data at a prespecified number of events $(15,31,49,98$, and 147) against efficacy (futility) boundaries derived on the basis of the alpha spending function approach. The trial did not halt for these assessments, and the trial team remained blinded to the study results until the final analysis.

PFS and OS were compared between study arms by using the log-rank test stratified by the baseline stratification factors, and corresponding twosided 95\% CIs were presented to align with the one-sided 2.5\% uppertailed test. Significant factors in univariable Cox proportional hazards regression analysis for OS were included in a multivariable Cox model to identify significant prognostic variables.

\section{Prognostic Value of HER1 and HER2 in the Screened Population}

Outcomes from the entire screening population were assessed to determine the prognostic value of HER1 or HER2 and to construct a prognostic 


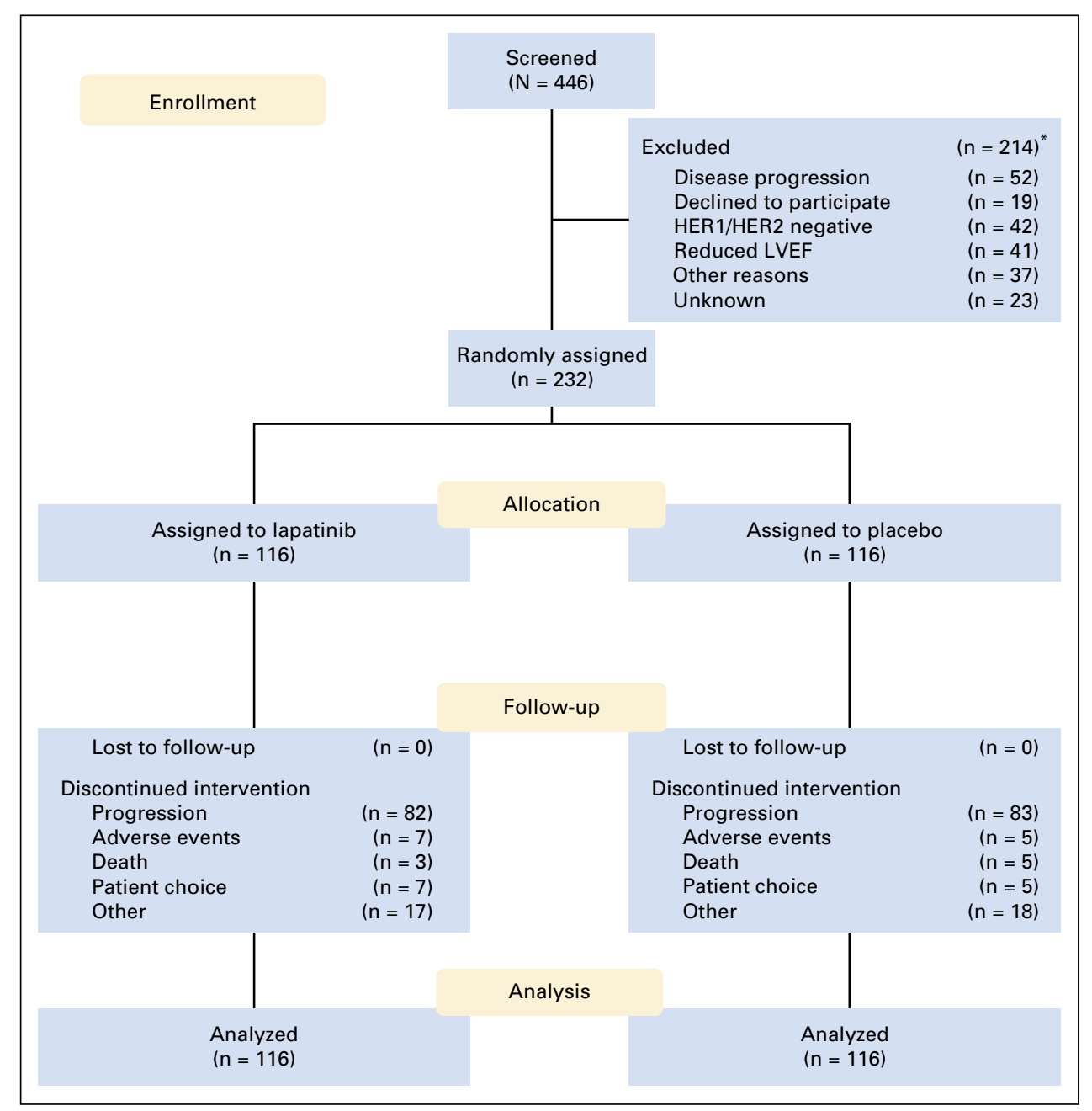

Fig 1. CONSORT diagram. Overview of screened and randomly assigned patients. * Only one reason for exclusion was available on the case report forms. Some patients possibly had more than one reason for exclusion. HER, human epidermal growth factor receptor; LVEF, left ventricular ejection factor.

index. The baseline time point for this exploratory analysis of the screening population was the date of completion of chemotherapy. OS was defined as the time from completion of chemotherapy to death or most recent followup. To construct the prognostic index, significant factors on multivariable analysis were dichotomized and added together. The prognostic index further categorized patients as low risk, medium risk, or high risk based on risk factor presence.

\section{RESULTS}

\section{Screening Population}

Between 2007 and 2013, 446 patients were screened for HER1 and HER2 status (Table 1). Overall, 329 (74\%) patients were male, and the median age was 71 years (interquartile range, 64 to 77 years; Table 1). The median number of chemotherapy cycles was six (interquartile range, four to six cycles), 61\% received cisplatinbased chemotherapy, and $48 \%$ had visceral metastasis. Subsequently, 133 (30\%) patients received second-line chemotherapy.

The median duration from the time tissue was taken for diagnosis to screening consent was 5 months (Appendix Fig A1, online only). Archived tissue was histologically T1 in 11\%, T2 to T3 in 64\%, T4 in 22\%, and from nodal/metastatic sites in 3\%. HER1 and HER2 positivity did not change with increasing T stage (data not shown). Fifteen percent of screened patients were negative for HER1 and 2, whereas 39\%, 13\%, and 33\% were positive for HER1 only, HER2 only, and HER1 and HER2, respectively. No significant difference was found in OS in terms of HER status in the screened population ( $P=.35$; Appendix Fig A2, online only), which suggests that it is not a prognostic factor. The most common reasons for ineligibility for random assignment were disease progression $(n=52[24 \%])$, patient choice $(n=19[8 \%])$, and reduced LVEF ( $\mathrm{n}=41$ [19\%]; Fig 1).

\section{Characteristics and Outcomes of the Randomly Assigned Population}

No significant differences in characteristics in the screened or randomly assigned population were found, except that the randomly assignment population included only patients positive for HER1/HER2 and excluded those with progression of disease (Table 1). Two hundred thirty-two patients were assigned to lapatinib $(\mathrm{n}=116)$ or placebo $(\mathrm{n}=116)$. The PFS for lapatinib and placebo was 4.5 (95\% CI, 2.8 to 5.4) months with 99 events and 5.1 (95\% CI, 3.0 to 5.8 ) months with 102 events, respectively (HR, 1.07; $95 \% \mathrm{CI}, 0.81$ to $1.43 ; P=.63)$. The OS for lapatinib and placebo was 12.6 (95\% CI, 9.0 to 16.2 ) months with 80 deaths and 12.0 (95\% CI, 10.5 to 14.9 ) months with 82 deaths, respectively 


\begin{tabular}{|c|c|c|c|c|}
\hline \multirow[b]{2}{*}{ Characteristic } & \multirow{2}{*}{$\begin{array}{c}\text { Screened but Not Randomly Assigned, } \\
\text { No. (\%) }\end{array}$} & \multicolumn{2}{|c|}{$\begin{array}{c}\text { Randomly Assigned ( } \mathrm{n}=232) \text {, } \\
\text { No. }(\%)\end{array}$} & \multirow[b]{2}{*}{ Total, No. (\%) } \\
\hline & & Lapatinib & Placebo & \\
\hline No. of patients & 214 & 116 & 116 & 445 \\
\hline \multicolumn{5}{|l|}{ Sex } \\
\hline Female & 57 (26.6) & $28(24.1)$ & 32 (27.6) & $117(26.2)$ \\
\hline Male & 157 (73.4) & 88 (75.9) & $84(72.4)$ & 329 (73.8) \\
\hline Median age (IQR), years & $70.4(64.7-77.2)$ & $70.7(63.9-77.2)$ & $71.1(63.8-76.3)$ & $70.7(64.2-77.1)$ \\
\hline \multicolumn{5}{|l|}{ Performance status } \\
\hline 0 & $30(22.6)$ & $53(45.7)$ & $52(44.8)$ & $125(35.2)$ \\
\hline 1 & 79 (59.4) & $52(44.8)$ & $51(44.0)$ & $187(52.7)$ \\
\hline$>1$ & $24(18.1)$ & $11(9.5)$ & $13(11.5)$ & $43(12.1)$ \\
\hline \multicolumn{5}{|c|}{ Response to previous chemotherapy } \\
\hline $\mathrm{CR}$ or $\mathrm{PR}$ & $92(51.1)$ & $80(69.0)$ & $78(67.2)$ & $250(60.7)$ \\
\hline SD & $36(20.0)$ & $36(31.0)$ & 38 (32.8) & $110(26.7)$ \\
\hline PD & 52 (28.9) & $0(0.0)$ & $0(0.0)$ & 52 (12.6) \\
\hline \multicolumn{5}{|l|}{ Tumor grade } \\
\hline 1 or 2 & $16(9.3)$ & $4(4.0)$ & $4(4.0)$ & $24(6.4)$ \\
\hline 3 or 4 & $156(90.7)$ & $98(96.0)$ & $98(96.1)$ & 352 (93.7) \\
\hline \multicolumn{5}{|l|}{ Visceral metastasis } \\
\hline Yes & $77(46.1)$ & $60(53.6)$ & $47(43.1)$ & $214(48.0)$ \\
\hline No & 90 (53.9) & $52(46.4)$ & $62(56.9)$ & $232(52.0)$ \\
\hline \multicolumn{5}{|l|}{ HER status } \\
\hline HER1 positive & $73(34.1)$ & $53(45.7)$ & $49(42.2)$ & $175(39.2)$ \\
\hline HER2 positive & $18(8.4)$ & $21(18.1)$ & $21(18.1)$ & 60 (13.5) \\
\hline Both positive & 57 (26.6) & 42 (36.2) & $46(39.7)$ & 145 (32.5) \\
\hline HER negative & 66 (30.8) & $0(0.0)$ & $0(0.0)$ & 66 (14.8) \\
\hline \multicolumn{5}{|c|}{ Previous cisplatin-based chemotherapy } \\
\hline Yes & 114 (57.3) & $71(64.0)$ & $73(65.2)$ & $258(61.4)$ \\
\hline No & $85(42.7)$ & $40(36.0)$ & 39 (34.8) & 164 (38.9) \\
\hline \multicolumn{5}{|l|}{ Hemoglobin level } \\
\hline Normal & $17(9.2)$ & $31(28.4)$ & $26(22.4)$ & $74(18.1)$ \\
\hline Low & $168(90.8)$ & 78 (71.6) & 90 (77.6) & 336 (81.9) \\
\hline \multicolumn{5}{|l|}{ Albumin level } \\
\hline Normal & $157(88.7)$ & 108 (99.1) & 111 (97.4) & $376(94.0)$ \\
\hline Low & $20(11.3)$ & $1(0.9)$ & $3(2.6)$ & $24(6.0)$ \\
\hline \multicolumn{5}{|l|}{ Creatinine level } \\
\hline Normal & 77 (41.9) & $49(45.0)$ & 36 (31.6) & 162 (39.8) \\
\hline High & $107(58.1)$ & $60(55.0)$ & 78 (68.4) & $245(60.2)$ \\
\hline
\end{tabular}

(HR, 0.96; 95\% CI, 0.70 to $1.31 ; P=.80$; Figs $2 \mathrm{~A}$ and $2 \mathrm{~B}$ ). CIs are wide because of the modest size of the trial. The best response rate for lapatinib and placebo were $14 \%$ versus $8 \%(P=.14)$.

Predefined subset analysis of patients positive for HER1/HER2 $3+$ on IHC $(n=111[48 \%])$, those positive for HER1 only $(n=102$ [44\%]), and those positive for HER2 only $(n=42[18 \%])$ showed no significant benefit in PFS (HRs, 0.90 [95\% CI, 0.59 to 1.36 ] with 101 events, 0.98 [95\% CI, 0.72 to 1.35 ] with 164 events, and 1.27 [95\% CI, 0.87 to 1.85 ] with 116 events, respectively; $P>.05$ for each) or OS (HRs, 0.77 [95\% CI, 0.48 to 1.24 ] with 81 deaths, 0.90 [95\% CI, 0.63 to 1.28 ] with 130 deaths, and 1.06 [95\% CI, 0.69 to 1.62] with 90 deaths, respectively; $P>.05$ for each) for lapatinib. Subgroup forest plot analysis also did not show a subgroup of patients who benefited from therapy (Appendix Fig A3, online only).

\section{Dose Reduction and AE Profile}

Lapatinib dose was reduced in 17 (7\%) patients. Discontinuation due to AEs was similar in both arms (6\% lapatinib and 5\% placebo). No significant difference was found in the frequency of AEs, which occurred in $>10 \%$ of patients (Table 2 ). The rate of grade 3 to 4 AEs for lapatinib and placebo was $8.6 \%$ versus $8.1 \%(P=.82)$.

\section{Prognosis of Patients at the Time of Completion of Chemotherapy}

The median OS for the screened population $(n=446)$ from the time of completion of chemotherapy was 11.8 (95\% CI, 10.0 to 12.9 ) months. Univariable analysis for survival was performed by using the screened population at the time of completion of chemotherapy (Table 3 ). Significant variables were included in the multivariable analysis, and results showed that poor performance status (HR, 1.53; 95\% CI, 1.28 to $1.84 ; P<.001$ ) and progression with chemotherapy (HR, 4.2; 95\% CI, 2.63 to 6.72; $P<.001)$ were associated with a poor OS. Visceral metastasis (HR, 1.32; 95\% CI, 1.01 to $1.71 ; P=.04$ ) was also significant. A prognostic index that incorporated these factors was generated. Figure 3 shows the survival of the three prognostic groups within this prognostic index. The 1-year OS for low-, medium-, and high-risk patients was $61.2 \%$ (95\% CI, $52.4 \%$ to $68.9 \%$ ), $49.1 \%$ 


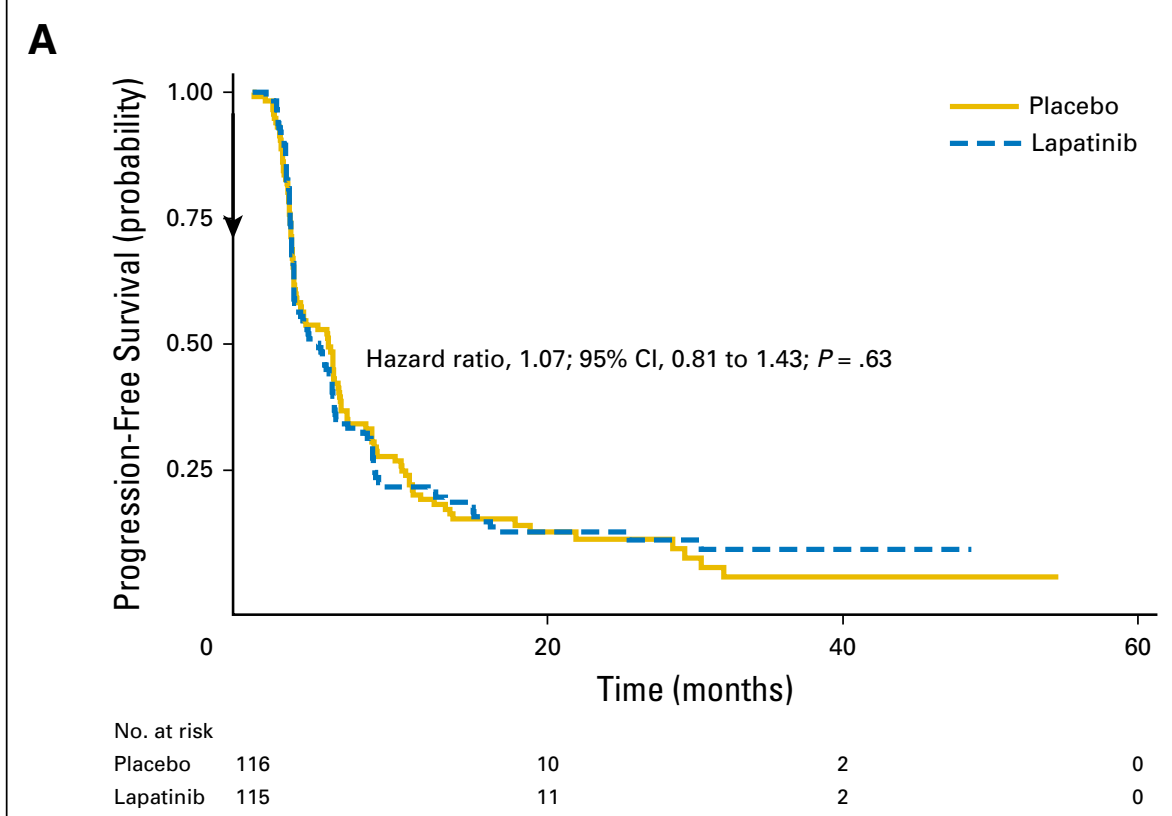

B

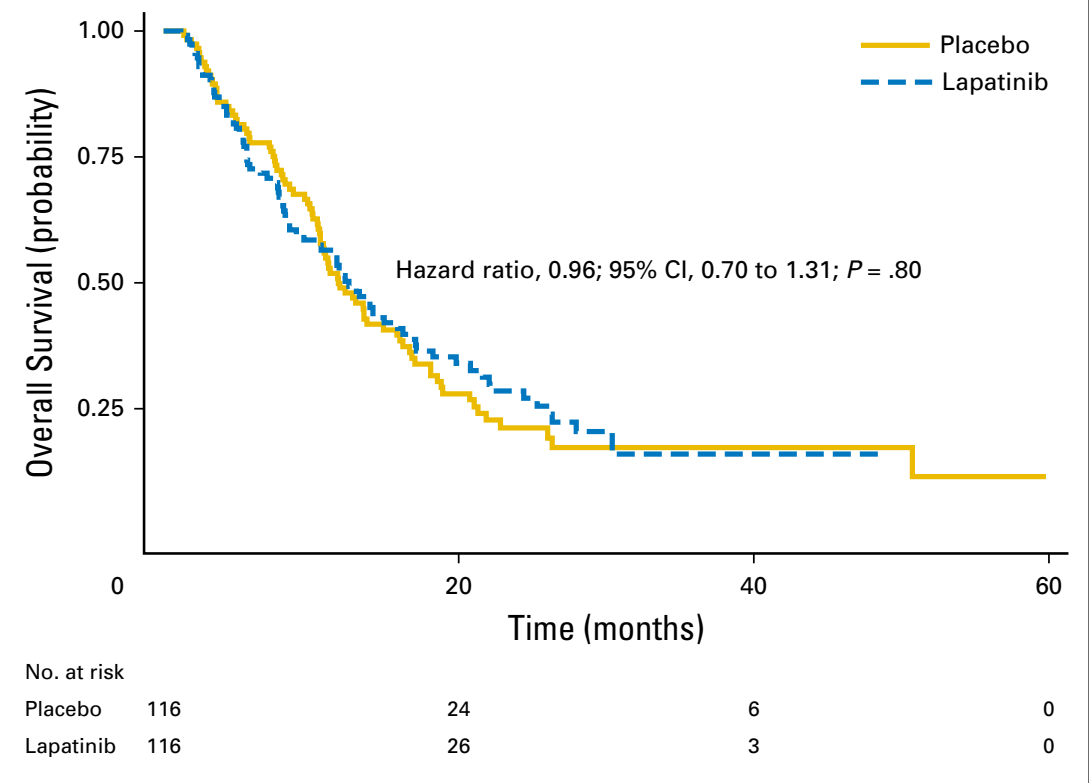

Fig 2. Comparison of outcomes for the randomly assigned population by Kaplan-Meier method. (A) Progression-free survival (the primary end point). (B) Overall survival (the secondary end point).
( $95 \% \mathrm{CI}, 40.7 \%$ to $57.1 \%$ ), and $21.9 \%$ (95\% CI, $12.7 \%$ to $32.6 \%$ ), respectively.

\section{DISCUSSION}

Maintenance lapatinib was not associated with clinical benefit in patients with HER1- and HER2-positive bladder cancer tumors as measured by our biomarker assay. Further analysis of subsets of patients positive for HER1 or HER2 did not show significant benefit associated with the drug, even in those tumors that expressed the highest level of the biomarker $\left(3+\right.$ on $\left.\mathrm{IHC}^{15}\right)$, which reinforces the lack of benefit. To our knowledge, this study is the first randomized phase III therapy trial in metastatic UBC to enrich for biomarkers and to use a maintenance design. The phase II results with lapatinib were worthy of further investigation in biomarker-positive patients with UBC previously treated with chemotherapy, ${ }^{15}$ which justified the current study. However, our strategy was unsuccessful for a number of possible reasons. First, although the targeting of HER proteins in isolation in breast cancer has been successful, they may not be a target associated with clinical benefit in UBC. ${ }^{8,19}$ Recent studies that investigated trastuzumab (HER2 antibody) with chemotherapy in UBC with HER2 gene amplification also do not support this theory. ${ }^{19}$ Different rates of biomarker positivity are seen with different methodologies, which highlights the 


\begin{tabular}{|c|c|c|c|c|}
\hline \multirow[b]{2}{*}{ Adverse Event } & \multicolumn{2}{|c|}{$\begin{array}{c}\text { Lapatinib }(n=97) \text {, No. } \\
(\%)\end{array}$} & \multicolumn{2}{|c|}{$\begin{array}{c}\text { Placebo }(n=99), \text { No. } \\
(\%)\end{array}$} \\
\hline & Grade 1-2 & Grade 3-4 & Grade 1-2 & Grade 3-4 \\
\hline Anorexia & $12(12.4)$ & $0(0.0)$ & $7(7.1)$ & $0(0.0)$ \\
\hline Constipation & $14(14.4)$ & $2(2.1)$ & $17(17.2)$ & $1(1.0)$ \\
\hline Cough & $8(8.2)$ & $0(0.0)$ & $9(9.1)$ & $1(1.0)$ \\
\hline Diarrhea & 59 (60.8) & $6(6.2)$ & $22(22.2)$ & $1(1.0)$ \\
\hline Fatigue & $34(35.1)$ & $4(4.1)$ & $41(41.4)$ & $1(1.0)$ \\
\hline Infection & $26(26.8)$ & $5(5.2)$ & $14(14.1)$ & $4(4.0)$ \\
\hline Itch & $12(12.4)$ & $0(0.0)$ & $11(11.1)$ & $1(1.0)$ \\
\hline Nausea & $22(22.7)$ & $1(1.0)$ & $19(19.2)$ & $1(1.0)$ \\
\hline Neuropathy & $7(7.2)$ & $0(0.0)$ & $13(13.1)$ & $1(1.0)$ \\
\hline Pain & $37(38.1)$ & $10(10.3)$ & $41(41.4)$ & $6(6.1)$ \\
\hline Rash & $43(44.3)$ & $2(2.1)$ & $21(21.2)$ & $0(0.0)$ \\
\hline Shortness of breath & $12(12.4)$ & $0(0.0)$ & $7(7.1)$ & $3(3.0)$ \\
\hline Vomiting & $15(15.5)$ & $3(3.1)$ & $15(15.2)$ & $1(1.0)$ \\
\hline
\end{tabular}

uncertainty around biomarker selection in this setting. Second, archived paraffin-embedded tissue was used to measure biomarker expression, which may not have been representative of current biomarker status. In addition, cancer tissue consisted largely of tissue from the bladder which may not be representative of metastatic disease. Third, the method of biomarker assessment (IHC and FISH analyses), which has been used successfully in breast cancer, ${ }^{20}$ may not be an effective approach in UBC. A spectrum of various IHC methodologies have been used to assess HER expression across different cancers. ${ }^{21}$ Our biomarker selection may be partly responsible for the results. Gene expression or amplification methodologies may be preferable in UBC. Fourth, the trial had an ambitious design by testing for a large clinical benefit in an enriched population. The CIs, therefore, are wide, which means that modest, but clinically meaningful differences were not detectable. Finally, although lapatinib has activity in other HER-positive cancers, it may not have activity in UBC, and other methods of targeting the HER family may be preferable. A recent phase II study in UBC with afatinib (an ERBB family inhibitor) showed promising activity. ${ }^{22}$ Therefore, activity that occurs with a different drug or biomarker is conceivable.

The maintenance trial design in this setting has not been used in previous randomized phase III studies in UBC, although other smaller studies that investigated sunitinib and vinflunine have been reported. ${ }^{9,23}$ The study with sunitinib showed short PFS and OS after chemotherapy, whereas the results with vinflunine suggested possible clinical activity. A new generation of immune therapy studies currently uses this trial design (NCT02500121).

Results from the screened population show that HER1 or HER2 status is not prognostic (Appendix Fig A2). This is the most robust analysis of this issue to our knowledge, which sheds light on previously contradictory data and increases our understanding of this complex area complicated by the various methodologies used to measure HER1 and HER2. ${ }^{13,14,16,18,24,25}$ Different methods of molecular analysis are possibly responsible for these contradictory results. Further work is required to determine whether HER1 or HER2 plays a role in UBC. ${ }^{13,14}$ This trial offered the opportunity to

\begin{tabular}{|c|c|c|c|c|c|c|c|c|c|c|}
\hline & \multicolumn{5}{|c|}{ PFS } & \multicolumn{5}{|c|}{ OS } \\
\hline & No. of Patients & Events & $H R$ & $95 \% \mathrm{Cl}$ & $P$ & No. of Patients & Events & $\mathrm{HR}$ & $95 \% \mathrm{Cl}$ & $P$ \\
\hline Age & 353 & 313 & 0.99 & 0.98 to 1.01 & .31 & 393 & 313 & 1.00 & 0.99 to 1.01 & .87 \\
\hline \multicolumn{11}{|l|}{ Sex } \\
\hline Female & 101 & 92 & 1 & - & - & 106 & 93 & 1 & - & - \\
\hline Male & 252 & 221 & 0.82 & 0.64 to 1.05 & .12 & 287 & 220 & 0.82 & 0.65 to 1.05 & .12 \\
\hline ECOG PS & 310 & 274 & 1.25 & 1.06 to 1.48 & .01 & 336 & 261 & 1.55 & 1.32 to 1.83 & $<.001$ \\
\hline \multicolumn{11}{|l|}{ HER status } \\
\hline HER negative & 44 & 41 & 1 & - & - & 47 & 43 & 1 & & \\
\hline HER1 positive & 141 & 121 & 1.21 & 0.85 to 1.72 & .30 & 158 & 125 & 1.07 & 0.75 to 1.51 & .72 \\
\hline HER2 positive & 53 & 48 & 1.25 & 0.82 to 1.90 & .29 & 58 & 48 & 0.90 & 0.59 to 1.36 & .62 \\
\hline Both positive & 115 & 103 & 1.00 & 0.70 to 1.44 & .99 & 130 & 97 & 0.84 & 0.59 to 1.21 & .36 \\
\hline \multicolumn{11}{|l|}{ Visceral metastasis } \\
\hline No & 176 & 151 & 1 & - & - & 184 & 137 & 1 & - & - \\
\hline Yes & 146 & 133 & 1.27 & 1.01 to 1.61 & .044 & 169 & 140 & 1.44 & 1.14 to 1.83 & .002 \\
\hline \multicolumn{11}{|c|}{ Response to previous chemotherapy } \\
\hline $\mathrm{CR}$ or $\mathrm{PR}$ & 235 & 209 & 1 & - & - & 230 & 184 & 1 & - & - \\
\hline SD & 110 & 98 & 1.03 & 0.81 to 1.31 & .83 & 109 & 78 & 0.94 & 0.72 to 1.23 & .67 \\
\hline PD & - & - & - & - & - & 47 & 46 & 3.06 & 2.20 to 4.26 & $<.001$ \\
\hline \multicolumn{11}{|l|}{ Tumor grade } \\
\hline 1 and 2 & 16 & 13 & 1 & - & - & 21 & 15 & 1 & - & - \\
\hline 3 and 4 & 290 & 257 & 1.10 & 0.63 to 1.92 & .75 & 318 & 253 & 1.31 & 0.78 to 2.20 & .32 \\
\hline \multicolumn{11}{|c|}{ Previous cisplatin-based chemotherapy } \\
\hline No & 137 & 126 & 1 & - & - & 148 & 122 & 1 & - & - \\
\hline Yes & 206 & 177 & 0.81 & 0.65 to 1.02 & .07 & 235 & 183 & 0.81 & 0.64 to 1.02 & .07 \\
\hline Hemoglobin level & 337 & 298 & 1.00 & 0.99 to 1.01 & .75 & 374 & 296 & 1.00 & 0.99 to 1.01 & .64 \\
\hline Albumin level & 331 & 293 & 0.97 & 0.94 to 0.99 & .01 & 366 & 289 & 0.92 & 0.89 to 0.94 & $<.001$ \\
\hline Creatinine level & 334 & 295 & 0.998 & 0.996 to 1.001 & .153 & 371 & 293 & 0.998 & 0.996 to 1.001 & .158 \\
\hline
\end{tabular}




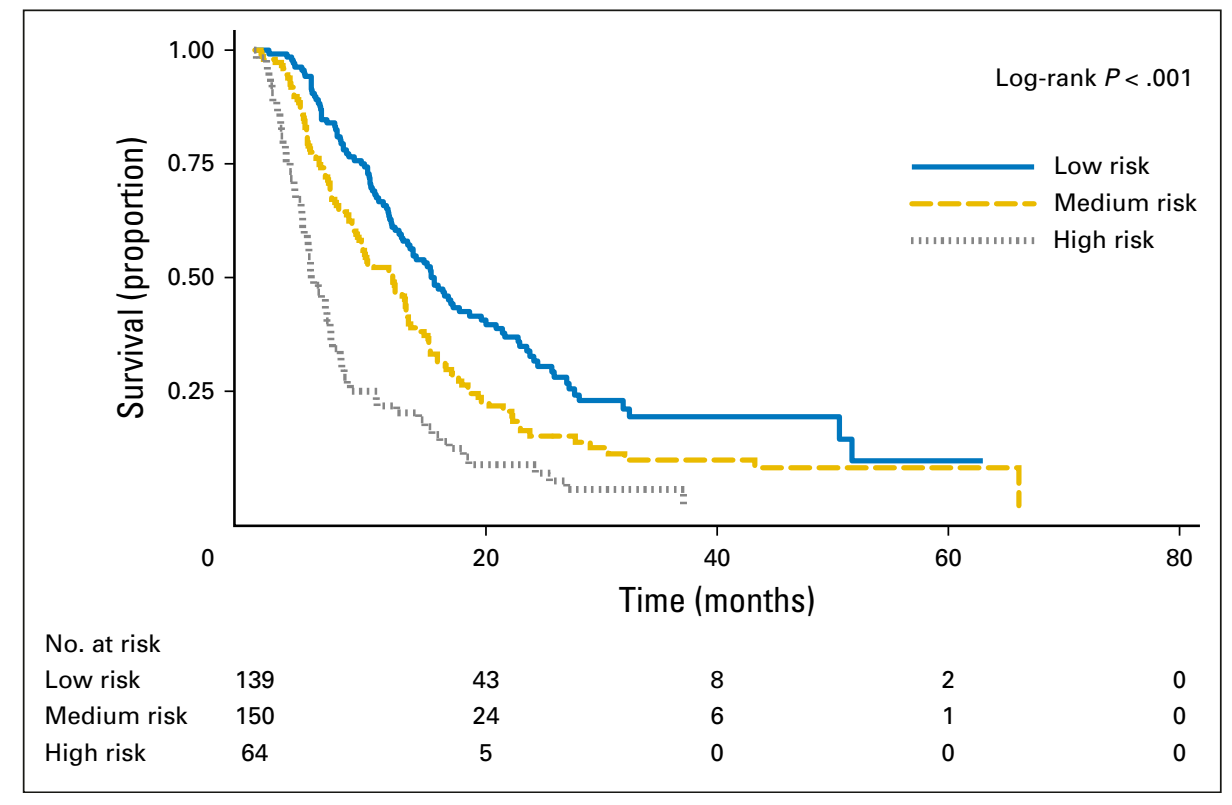

Fig 3. Prognostic index that predicts outcome from the time of chemotherapy completion. The last day of chemotherapy was considered the start date for data analysis. Overall survival was the chosen end point. Factors associated with a poor outcome on multivariable analysis were included in the prognostic model: Low performance status, progression of disease on chemotherapy, and presence of visceral metastasis were all allocated 1 point. Three groups (low risk = no factors; medium risk $=$ one factor; high risk = more than one factor) were formed. explore the importance of gene expression in unselected patients, but we focused predominately on protein expression. Future research in this area would be helpful.

The trial design allowed us to study the characteristics and outcomes of unselected patients after the completion of chemotherapy, which has not been reported previously. More than one half $(61 \%)$ of the patients received cisplatin, and a high proportion (48\%) had visceral metastasis, which gives some insight into the current population of patients who receive chemotherapy. Survival was short after the completion of chemotherapy ( $<1$ year) in both the randomly assigned and screened populations, which underscores the poor outcome for patients with metastatic UBC, particularly those not eligible for cisplatin chemotherapy. ${ }^{1}$ Only $30 \%$ of patients received second-line chemotherapy (29 in the lapatinib arm and 34 in the placebo arm; $P=.49$ ), which may have contributed to this finding. Together, these results show that patients with UBC have a poor outcome, even if they initially gained clinical benefit from chemotherapy. ${ }^{25}$ Whether the patients in this study had a less-thanexpected good outcome remains largely unknown because of the paucity of comparative data in this setting.

Prognostic factors were also assessed in this population. Previous studies of prognostic factors focused on clinical parameters before the start of first-line therapy. ${ }^{26}$ The current trial design allowed us to analyze prognostic factors at the time of completion of chemotherapy. Results showed that radiologic progression on chemotherapy, visceral metastasis, and poor performance status were associated with a poor outcome in multivariable analysis. A prognostic model that consisted of these factors was constructed and discriminated patients into three groups. Although further validation is required, this information is novel and helpful to patients and their caregivers.

The trial design was pragmatic and allowed for a varying number of chemotherapy cycles and regimens. Previous perioperative therapy was not an exclusion criterion, and the proportion of patients who received this was not recorded. In addition, patients could be randomly assigned up to 10 weeks after chemotherapy. Although this makes the study applicable to a broad spectrum of patients, it may introduce bias. Results show that the median number of chemotherapy cycles and the use of cisplatin were similar in both random assignment groups, which alleviates some of these concerns. However, differences in the quality of responses between cisplatin and carboplatin are a concern. Indeed, a significant proportion of patients who received placebo had continued response, which suggests ongoing activity of chemotherapy beyond the last dose administered.

Despite these shortcomings, this study shows that lapatinib does not significantly improve outcomes in this subset of patients with UBC. Further exploration with different agents and different biomarkers continue. A more detailed understanding of the role of HER1 and HER2 in UBC should be pursued in future trials.

\section{AUTHORS' DISCLOSURES OF POTENTIAL CONFLICTS} OF INTEREST

Disclosures provided by the authors are available with this article at ascopubs.org/journal/jco.

\section{AUTHOR CONTRIBUTIONS}

Conception and design: Thomas Powles, Shah-Jalal Sarker, Simon Chowdhury

Provision of study materials or patients: Robert A. Huddart, Simon Crabb, Simon Chowdhury

Collection and assembly of data: Thomas Powles, Robert A. Huddart, Tony Elliott, Charlotte Ackerman, Robert Jones, Syed Hussain, Simon Crabb, Satinder Jagdev, John Chester, Serena Hilman, Mark Beresford, Graham Macdonald, Sundar Santhanam, John A. Frew, Andrew Stockdale, Simon Hughes, Daniel Berney, Simon Chowdhury

Data analysis and interpretation: Thomas Powles, Robert A. Huddart, Shah-Jalal Sarker, Charlotte Ackerman, Daniel Berney, Simon Chowdhury Manuscript writing: All authors

Final approval of manuscript: All authors 


\section{REFERENCES}

1. De Santis M, Bellmunt $J$, Mead $G$, et al: Randomized phase I//III trial assessing gemcitabine/ carboplatin and methotrexate/carboplatin/vinblastine in patients with advanced urothelial cancer who are unfit for cisplatin-based chemotherapy: EORTC study 30986. J Clin Oncol 30:191-199, 2012

2. Bellmunt J, von der Maase $\mathrm{H}$, Mead GM, et al: Randomized phase III study comparing paclitaxel/ cisplatin/ gemcitabine and gemcitabine/cisplatin in patients with locally advanced or metastatic urothelial cancer without prior systemic therapy: EORTC intergroup study 30987. J Clin Oncol 30:1107-1113, 2012

3. Bellmunt $J$, Théodore $C$, Demkov $T$, et al: Phase III trial of vinflunine plus best supportive care compared with best supportive care alone after a platinum-containing regimen in patients with advanced transitional cell carcinoma of the urothelial tract. J Clin Oncol 27:4454-4461, 2009 [Erratum: $\mathrm{J}$ Clin Oncol 28:182, 2010]

4. Kopparapu PK, Boorjian SA, Robinson BD, et al: Expression of VEGF and its receptors VEGFR1/ VEGFR2 is associated with invasiveness of bladder cancer. Anticancer Res 33:2381-2390, 2013

5. McHugh LA, Sayan AE, Mejlvang J, et al: Lapatinib, a dual inhibitor of ErbB-1/-2 receptors, enhances effects of combination chemotherapy in bladder cancer cells. Int J Oncol 34:1155-1163, 2009

6. McHugh LA, Kriajevska M, Mellon JK, et al: Combined treatment of bladder cancer cell lines with lapatinib and varying chemotherapy regimensevidence of schedule-dependent synergy. Urology 69:390-394, 2007

7. Hussain M, Daignault $S$, Agarwal N, et al: A randomized phase 2 trial of gemcitabine/cisplatin with or without cetuximab in patients with advanced urothelial carcinoma. Cancer 120:2684-2693, 2014

8. Choueiri TK, Ross RW, Jacobus $S$, et al: Double-blind, randomized trial of docetaxel plus vandetanib versus docetaxel plus placebo in platinum- pretreated metastatic urothelial cancer. J Clin Oncol 30:507-512, 2012

9. Grivas PD, Daignault S, Tagawa ST, et al: Double-blind, randomized, phase 2 trial of maintenance sunitinib versus placebo after response to chemotherapy in patients with advanced urothelial carcinoma. Cancer 120:692-701, 2014

10. Galsky MD, Hahn NM, Powles T, et al: Gemcitabine, cisplatin, and sunitinib for metastatic urothelial carcinoma and as preoperative therapy for muscle-invasive bladder cancer. Clin Genitourin Cancer 11:175-181, 2013

11. Culine S, Sellam Z, Bouaita $L$, et al: Combining paclitaxel and lapatinib as second-line treatment for patients with metastatic transitional cell carcinoma: A case series. Anticancer Res 32:3949-3952, 2012

12. Kandoth $\mathrm{C}$, McLellan MD, Vandin $\mathrm{F}$, et al: Mutational landscape and significance across 12 major cancer types. Nature 502:333-339, 2013

13. Ding $W$, Tong S, Gou Y, et al: Human epidermal growth factor receptor 2: A significant indicator for predicting progression in non-muscle-invasive bladder cancer especially in high-risk groups. World J Urol 33: 1951-1957, 2015

14. Tsai YS, Cheng HL, Tzai TS, et al: Clinical significance of ErbB receptor family in urothelial carcinoma of the bladder: A systematic review and meta-analysis. Adv Urol 2012:181964, 2012

15. Wülfing C, Machiels JP, Richel DJ, et al: A single-arm, multicenter, open-label phase 2 study of lapatinib as the second-line treatment of patients with locally advanced or metastatic transitional cell carcinoma. Cancer 115:2881-2890, 2009

16. Hussain MHA, MacVicar GR, Petrylak DP, et al: Trastuzumab, paclitaxel, carboplatin, and gemcitabine in advanced human epidermal growth factor receptor-2/neu-positive urothelial carcinoma: Results of a multicenter phase II National Cancer Institute trial. J Clin Oncol 25:2218-2224, 2007

17. Gårdmark $T$, Wester $K$, De la Torre $M$, et al: Analysis of HER2 expression in primary urinary bladder carcinoma and corresponding metastases. BJU Int 95:982-986, 2005
18. Jimenez RE, Hussain M, Bianco FJ Jr, et al: Her-2/neu overexpression in muscle-invasive urothelial carcinoma of the bladder: Prognostic significance and comparative analysis in primary and metastatic tumors. Clin Cancer Res 7:2440-2447, 2001

19. Oudard S, Culine S, Vano $Y$, et al: Multicentre randomised phase II trial of gemcitabine+platinum, with or without trastuzumab, in advanced or metastatic urothelial carcinoma overexpressing Her2. Eur J Cancer 51:45-54, 2015

20. Cameron D, Casey $M$, Press $M$, et al: A phase III randomized comparison of lapatinib plus capecitabine versus capecitabine alone in women with advanced breast cancer that has progressed on trastuzumab: Updated efficacy and biomarker analyses. Breast Cancer Res Treat 112:533-543, 2008

21. Rüschoff J, Hanna W, Bilous $M$, et al: HER2 testing in gastric cancer: A practical approach. Mod Pathol 25:637-650, 2012

22. Choudhury NJ, Campanile A, Antic T, et al: Afatinib activity in platinum-refractory metastatic urothelial carcinoma in patients with ERBB alterations. J Clin Oncol 34:2165-2171, 2016

23. Bellmunt J, Perez Valderrama $B$, Font $A$, et al: Maintenance vinflunine post cisplatin chemotherapy (CT) in patients with advanced urothelial carcinoma (UC): Preliminary analysis of a randomized placebo controlled phase II trial (MAJA trial)-SOGUG 201102. J Clin Oncol 33, 2015 (suppl; abstr 4529)

24. Chow NH, Chan SH, Tzai TS, et al: Expression profiles of ErbB family receptors and prognosis in primary transitional cell carcinoma of the urinary bladder. Clin Cancer Res 7:1957-1962, 2001

25. Memon AA, Sorensen BS, Meldgaard $P$, et al: The relation between survival and expression of HER1 and HER2 depends on the expression of HER3 and HER4: A study in bladder cancer patients. $\mathrm{Br} \mathrm{J}$ Cancer 94:1703-1709, 2006

26. Powles T, Eder JP, Fine GD, et al: MPDL3280A (anti-PD-L1) treatment leads to clinical activity in metastatic bladder cancer. Nature 515:558-562, 2014

\section{Affiliations}

Thomas Powles, Shah-Jalal Sarker, Charlotte Ackerman, and Daniel Berney, Queen Mary University of London; Simon Hughes and Simon Chowdhury, Guy's and St Thomas' National Health Service (NHS) Foundation Trust, London; Robert A. Huddart, Institute of Cancer Research, Sutton; Tony Elliott, Christie Hospital NHS Foundation Trust, Manchester; Robert Jones, University of Glasgow, Glasgow; Syed Hussain, University of Liverpool, Liverpool; Simon Crabb, University of Southampton, Southampton; Satinder Jagdev, St James's University Hospital, Leeds; John Chester, Cardiff University, Cardiff; Serena Hilman, Bristol Haematology and Oncology Centre, Bristol; Mark Beresford, Royal United Hospitals Bath, Bath; Graham Macdonald, Aberdeen Royal Infirmary, Aberdeen; Sundar Santhanam, Nottingham University Hospitals NHS Trust, Nottingham; John A. Frew, Northern Centre for Cancer Care, Newcastle upon Tyne; and Andrew Stockdale, University Hospital, Coventry, United Kingdom.

\section{Support}

Supported by a GlaxoSmithKline research grant and the Cancer Research UK Experimental Cancer Medicine Centre. 
Phase III, Double-Blind, Randomized Trial That Compared Maintenance Lapatinib Versus Placebo After First-Line Chemotherapy in Patients With Human Epidermal Growth Factor Receptor 1/2-Positive Metastatic Bladder Cancer

The following represents disclosure information provided by authors of this manuscript. All relationships are considered compensated. Relationships are self-held unless noted. I = Immediate Family Member, Inst = My Institution. Relationships may not relate to the subject matter of this manuscript. For more information about ASCO's conflict of interest policy, please refer to www.asco.org/rwc or ascopubs.org/jco/site/ifc.

Thomas Powles

Honoraria: Roche, Genentech, Bristol-Myers Squibb, Novartis, GlaxoSmithKline

Consulting or Advisory Role: Roche, Genentech, Bristol-Myers Squibb, Merck, GlaxoSmithKline

Research Funding: AstraZeneca, MedImmune, Roche, Genentech, GlaxoSmithKline

Robert A. Huddart

Leadership: Cancer Centre London

Honoraria: Roche, Merck Sharp \& Dohme, Astellas Pharma, Janssen Pharmaceuticals

Research Funding: Merck Sharp \& Dohme (Inst)

Travel, Accommodations, Expenses: Merck Sharp \& Dohme, Janssen-Cilag

Tony Elliott

Research Funding: Bayer AG (Inst), Astellas Pharma (Inst), Orion Pharma (Inst), AstraZeneca (Inst), Janssen Pharmaceuticals (Inst), Pierre Fabre

(Inst), Sanofi (Inst), Celgene (Inst)

Travel, Accommodations, Expenses: Astellas Pharma, Janssen

Pharmaceuticals

Shah-Jalal Sarker

No relationship to disclose

\section{Charlotte Ackerman}

No relationship to disclose

Robert Jones

Honoraria: GlaxoSmithKline

Speakers' Bureau: GlaxoSmithKline

Research Funding: GlaxoSmithKline

Syed Hussain

Consulting or Advisory Role: Pierre Fabre, Astellas Pharma, Bayer AG, Janssen-Cilag, Roche, Genentech, Merck, Ipsen

Research Funding: Boehringer Ingelheim, Janssen-Cilag, Pierre Fabre

Travel, Accommodations, Expenses: Janssen-Cilag, Bayer AG,

Boehringer Ingelheim, Pierre Fabre, Astellas Pharma, Pfizer, Roche,

Merck Sharp \& Dohme

\section{Simon Crabb}

Honoraria: Bayer AG, Astellas Pharma

Consulting or Advisory Role: Bayer AG, Sanofi, Astellas Pharma, Janssen Pharmaceuticals, Dendreon, Pfizer

Research Funding: AstraZeneca, Astex Pharmaceuticals

Travel, Accommodations, Expenses: Astellas Pharma, Sanofi, Novartis, Janssen Pharmaceuticals, Bayer AG

\section{Satinder Jagdev}

No relationship to disclose

\section{John Chester}

Honoraria: Glycotope

Consulting or Advisory Role: Glycotope

Research Funding: AstraZeneca

Travel, Accommodations, Expenses: Glycotope, Tiziana Life Sciences, Merck Sharp \& Dohme

\section{Serena Hilman}

Consulting or Advisory Role: Bayer AG

Speakers' Bureau: Pfizer

Research Funding: Merck Serono (Inst), Astellas Pharma (Inst) Travel, Accommodations, Expenses: Bayer AG

Mark Beresford

Honoraria: Roche, AstraZeneca, Eisai, Novartis

Research Funding: AstraZeneca

Travel, Accommodations, Expenses: Bayer AG

\section{Graham Macdonald}

No relationship to disclose

Sundar Santhanam

No relationship to disclose

John A. Frew

No relationship to disclose

Andrew Stockdale

No relationship to disclose

\section{Simon Hughes}

Honoraria: Sanofi, Janssen Pharmaceuticals, Astellas Pharma, Bayer AG Consulting or Advisory Role: Astellas Pharma, Janssen Pharmaceuticals Speakers' Bureau: Astellas Pharma, Bayer AG, Sanofi, Janssen Pharmaceuticals

Travel, Accommodations, Expenses: Astellas Pharma

\section{Daniel Berney}

Honoraria: Sanofi, Molinari Diagnostics

Research Funding: Myriad Genetics

\section{Simon Chowdhury}

Honoraria: Novartis, Janssen Pharmaceuticals, Sanofi, Bayer AG, GlaxoSmithKline

Consulting or Advisory Role: Novartis, Astellas Pharma, Janssen Pharmaceuticals, ESSA Pharma, Sanofi, Bayer AG, Clovis Oncology Speakers' Bureau: Astellas Pharma, GlaxoSmithKline, Janssen Pharmaceuticals, Bayer AG, Pfizer

Research Funding: Janssen Pharmaceuticals, Sanofi

Travel, Accommodations, Expenses: GlaxoSmithKline, Astellas Pharma, Janssen Pharmaceuticals, Bayer AG, Clovis Oncology 


\section{Acknowledgment}

We thank Kashfia Chowdhury for statistical input.

\section{Appendix}

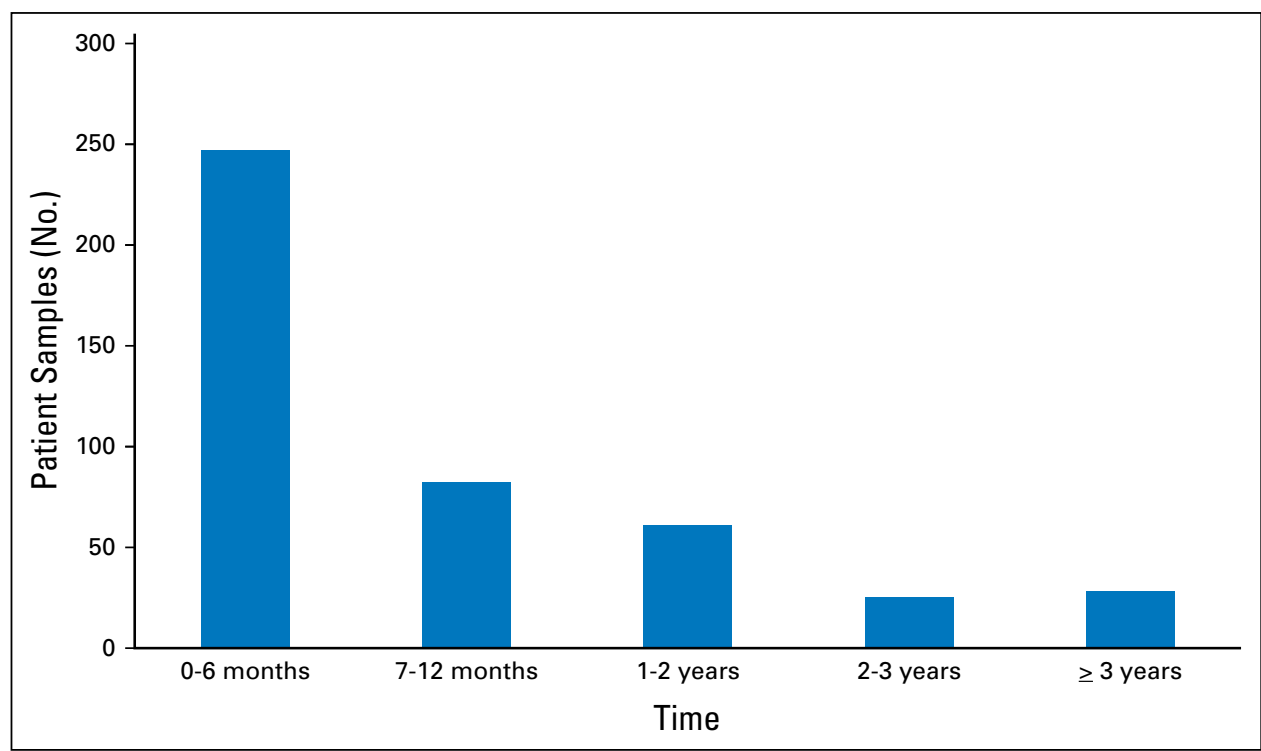

Fig A1. Time from tissue collection (archived formalin-fixed paraffin-embedded tissue) to screening.

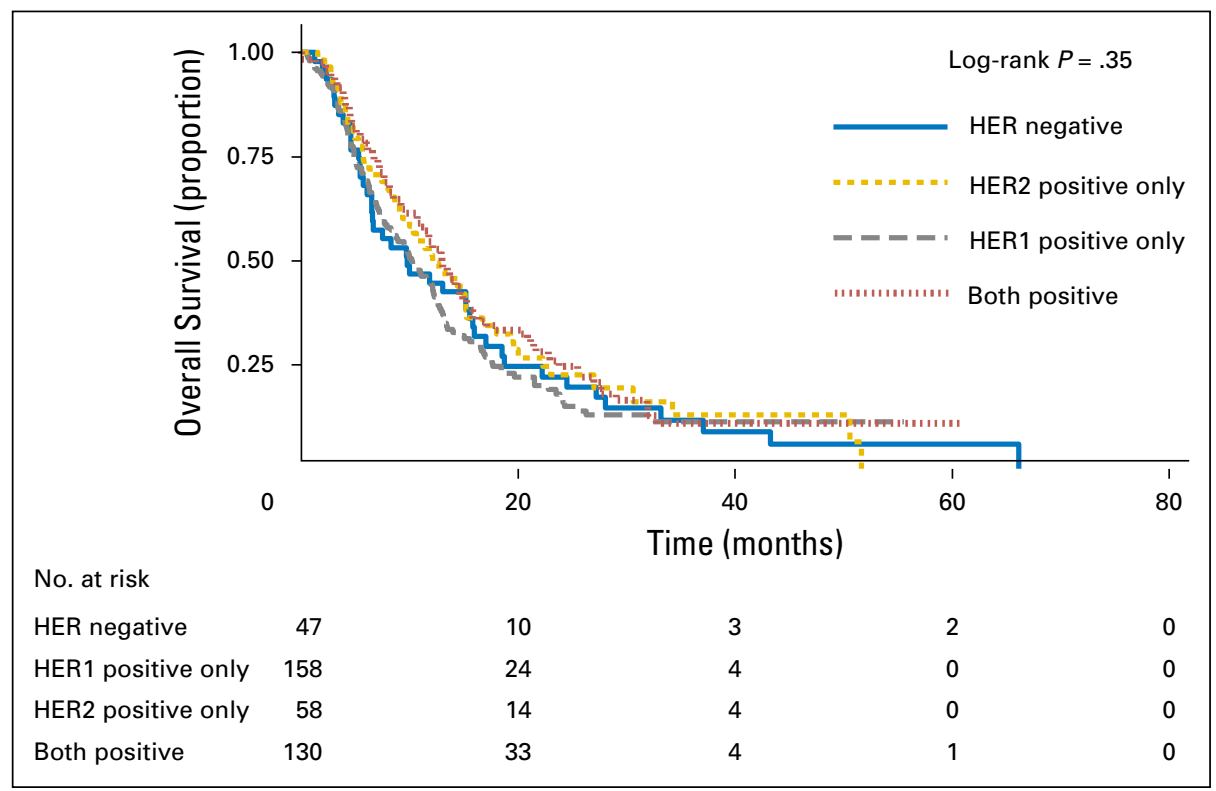

Fig A2. Kaplan-Meier overall survival curve by human epidermal growth factor receptor (HER) status from completion of chemotherapy. 


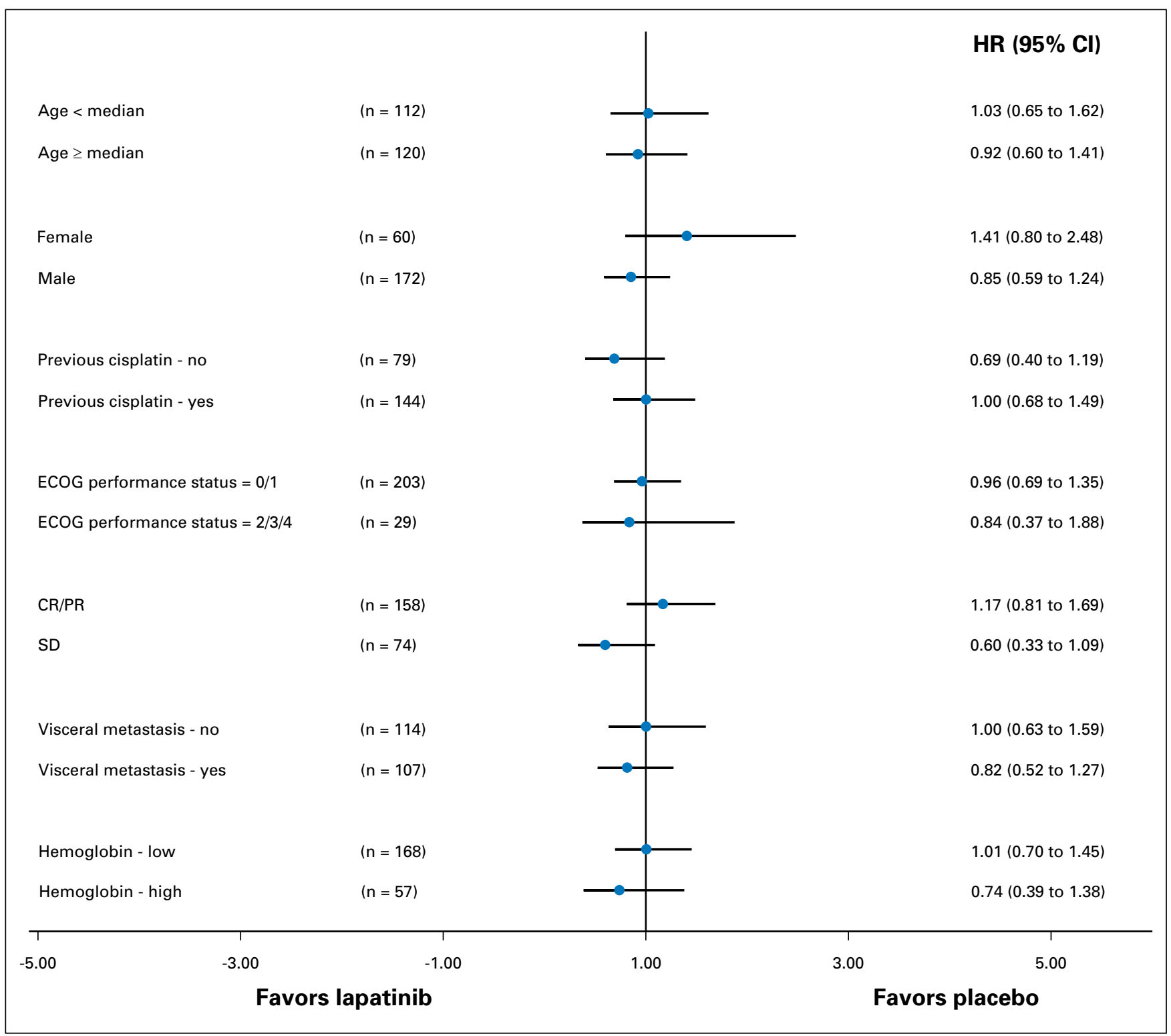

Fig A3. Subgroup forest plot analysis for overall survival. CR, complete response; ECOG, Eastern Cooperative Oncology Group; HR, hazard ratio; PR, partial response; $\mathrm{SD}$, stable disease 